
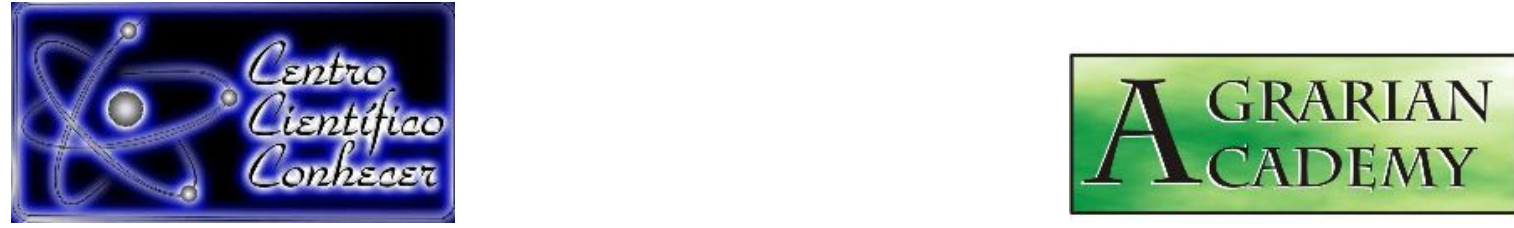

\title{
ASPECTOS SILVICULTURAIS DE Astronium gracile Engl.: SUBSÍDIOS PARA USO EM SISTEMAS AGROFLORESTAIS
}

\author{
Breno Pinto Rayol ${ }^{1}$; Fabrízia de Oliveira Alvino-Rayol ${ }^{2}$
}

1. Professor Doutor do Instituto de Ciências Agrárias da Universidade Federal

Rural da Amazônia, Belém, PA, Brasil (bprayol@yahoo.com.br).

2. Professora Doutora da Coordenação de Saneamento Ambiental do Instituto

Federal de Educação, Ciência e Tecnologia do Pará, Belém, PA, Brasil.

Recebido em: 19/11/2018 - Aprovado em: 14/12/2018 - Publicado em: 25/12/2018

DOI: 10.18677/Agrarian_Academy_2018B2

\begin{abstract}
O presente trabalho reúne informações silviculturais de Astronium gracile Engl., para subsidiar seu cultivo em sistemas agroflorestais. No laboratório foram conduzidas as análises de germinação e biometria das sementes. A avaliação do desenvolvimento das mudas transplantadas para uma floresta secundária foi realizada através de avaliações de sobrevivência e crescimento em altura. O tempo de germinação ocorre entre 13 a 15 dias com taxa de germinação média de 95,5\%. A taxa de sobrevivência foi de $70,8 \%$ em dois anos e o incremento periódico anual em altura de $37,2 \mathrm{~cm} / \mathrm{ano}$. Os resultados indicam que a espécie estudada apresentou desempenho aceitável quanto à sobrevivência e crescimento, reunindo características silviculturais promissoras para o uso em sistemas agroflorestais.
\end{abstract}

RESUMO

PALAVRAS-CHAVE: Agrossilvicultura, Amazônia, espécie florestal

\section{SILVICULTURE OF Astronium gracile Engl.: SUBSIDIES FOR USE IN AGROFORESTRY SYSTEMS}

\begin{abstract}
This paper presents the silvicultural of Astronium gracile Engl. provide subsidies for its cultivation in agroforestry systems. In the laboratory analyzes were conducted for seed germination and biometrics. The evaluation of the development of the seedlings transplanted into a secondary forest was conducted by evaluating survival and height growth. The germination time is between 13 and 15 days with an average germination rate of $95.5 \%$. The survival rate was $70.8 \%$ at two years and annual periodic increment in height of $37.2 \mathrm{~cm} /$ year. The results indicate that the species showed acceptable performance in terms of survival and growth. These silvicultural characteristics indicate that the species is promising for use in agroforestry systems.

KEYWORDS: Agroforestry, Amazon, forest species
\end{abstract}




\section{INTRODUÇÃO}

Astronium gracile Engl. pertencente à família Anacardiaceae, é uma espécie madeireira citada muito frequentemente em trabalhos de inventários florestais na região amazônica (COELHO, 2013; GUALBERTO et al., 2014; LEMOS et al., 2015; FERREIRA et al., 2015; SANTOS et al., 2018).

Apesar do conhecimento da silvicultura de espécies nativas ser importante para atividades de reflorestamento (MENDONÇA et al., 2017), recomposição das florestas ciliares (DO VALE, et al., 2014), restauração florestal (STOLARSKI et al., 2018) e sistemas de integração pecuária floresta (NIERI et al., 2017), ainda há escassez de informações silviculturais de espécies nativas com potencial para o mercado produtor (KLEIN et al., 2016).

Desta forma, o conhecimento da silvicultura e ecologia de espécies arbóreas é de grande importância para o êxito dos empreendimentos florestais. Apesar disso, ainda existem muitas lacunas sobre tais informações para a maioria das espécies arbóreas amazônicas. Portanto, o presente trabalho objetivou reunir informações silviculturais de Astronium gracile, com vistas a subsidiar e incentivar o cultivo em sistemas agroflorestais.

\section{MATERIAL E MÉTODOS}

No Laboratório de Sementes da Universidade Federal do Oeste do Pará (UFOPA), localizado no município de Santarém, foram conduzidas as análises de germinação, do grau de umidade e das características biométricas das sementes. As sementes de Astronium gracile utilizadas no presente estudo foram procedentes das matrizes localizadas na Estação Experimental de Curuá-Una (Prainha, PA). Os frutos foram coletados nas árvores com a utilização de podão e uma lona plástica para impedir o contato das sementes e frutos com o chão, reduzindo o risco de contaminação. Após a coleta dos frutos, efetuou-se a extração das sementes e em seguida a caracterização biométrica das mesmas.

O comprimento e a espessura das sementes foram determinados com auxílio de um paquímetro digital, sendo utilizadas 100 sementes. A determinação do grau de umidade foi realizada em quatro repetições de 10 sementes, adotando-se 0 método de estufa a $105 \pm 3^{\circ} \mathrm{C}$, durante 24 horas, de acordo com as Regras para Análise de Sementes (BRASIL, 2009). A determinação da massa de mil sementes foi realizada com oito repetições, procedendo-se conforme Brasil (2009).

Os testes de germinação foram conduzidos em ambiente controlado, (germinador à temperatura de $30^{\circ} \mathrm{C}$ ) em caixas plásticas transparentes $(11 \mathrm{~cm} \times$ $11 \mathrm{~cm} \times 3 \mathrm{~cm}$ ), contendo três diferentes substratos: a) (T1) areia lavada e esterilizada em estufa a $150^{\circ} \mathrm{C}$ por seis horas; b) (T2) vermiculita esterilizada e, c) (T3) terra preta esterilizada. As contagens das sementes germinadas foram feitas a cada três dias até o $30^{\circ}$ dia após à instalação do experimento, considerando-se como semente germinada toda aquela que deu origem a plântula normal. Foram consideradas as percentagens de plântulas anormais e de sementes mortas, as quais foram identificadas conforme Brasil (2009).

O cálculo de percentagem foi obtido pela fórmula citada em Labouriau (1983). $\mathrm{O}$ delineamento experimental foi o inteiramente casualizado com três tratamentos e cinco repetições de 25 sementes, totalizando 375 sementes. Para as análises estatísticas os dados de percentagem de germinação foram transformados em arc sen $(x / 100)^{0,5}$. Os dados foram submetidos à análise de variância, e as médias comparadas pelo teste de Tukey ao nível de $5 \%$ de probabilidade. 
A avaliação do desenvolvimento das mudas transplantadas para uma floresta secundária foi realizada em um fragmento florestal urbano de 1,4 ha em estágio inicial de sucessão, denominado de "Bosque Mekdece" pertencente à Universidade Federal do Oeste do Pará (UFOPA), município de Santarém, Oeste do Pará. Em Abril de 2009 foram transplantadas 27 mudas de aroeira, produzidas no Viveiro Florestal da UFOPA. As mudas foram distribuídas sistematicamente em cinco linhas com distância de $20 \mathrm{~m}$, com o espaçamento entre si de $2 \times 2 \mathrm{~m}$. A coleta de dados foi realizada através de avaliações de sobrevivência e crescimento em altura. A coleta da altura foi realizada com auxílio de uma trena graduada em centímetros.

\section{RESULTADOS E DISCUSSÃO}

O comprimento e o diâmetro médios das sementes de Astronium gracile foram de 10,5 mm e 1,7 mm, respectivamente. Em um quilograma de sementes contém cerca de 46.000 unidades. A germinação ocorre entre 13 e 15 dias, com a taxa de germinação média de $95,5 \%$, variando de $92 \%$ a $98 \%$.

Não foram observadas diferenças significativas entre os substratos na porcentagem de germinação de sementes de Astronium gracile (Tabela 1). A alta percentagem de germinação encontrada nos três tratamentos indicou que todos os substratos avaliados foram eficientes na germinação das sementes da espécie estudada. Os resultados obtidos no presente estudo estão de acordo com Santos et al. (2015) e Souza et al. (2012) que não constataram a influência de diferentes substratos na germinação de outras espécies do mesmo gênero da avaliada no presente estudo (Astronium graveolens e Astronium concinnum, respectivamente)

TABELA 1. Efeito dos diferentes substratos sobre o percentual de germinação de sementes de Astronium gracile.

\begin{tabular}{lc}
\hline \multicolumn{1}{c}{ Tratamentos (Substratos) } & Germinação (\%) \\
\hline Areia & $97,0 \%$ a \\
Terra preta & $94.0 \%$ a \\
Vermiculita & $96,0 \%$ a \\
\hline Médias seguidas da mesma letra na coluna não diferem \\
estatisticamente pelo teste Tukey ( $p \leq 0,05)$. \\
Fonte: Autores (2018)
\end{tabular}

A taxa de sobrevivência das mudas de Astronium gracile foi de $70,8 \%$ no período de 24 meses, não sendo observados ataques graves de insetos e/ou fitopatógenos nas mudas transplantadas. As mudas obtiveram um incremento periódico anual em altura de $37,2 \mathrm{~cm} /$ ano e aos 24 meses altura média de $110 \mathrm{~cm}$ (Figura 1) apresentando, desta forma, um bom desempenho em ambiente com certo grau de sombreamento, como é o caso da floresta secundária onde foi implantado o experimento. 


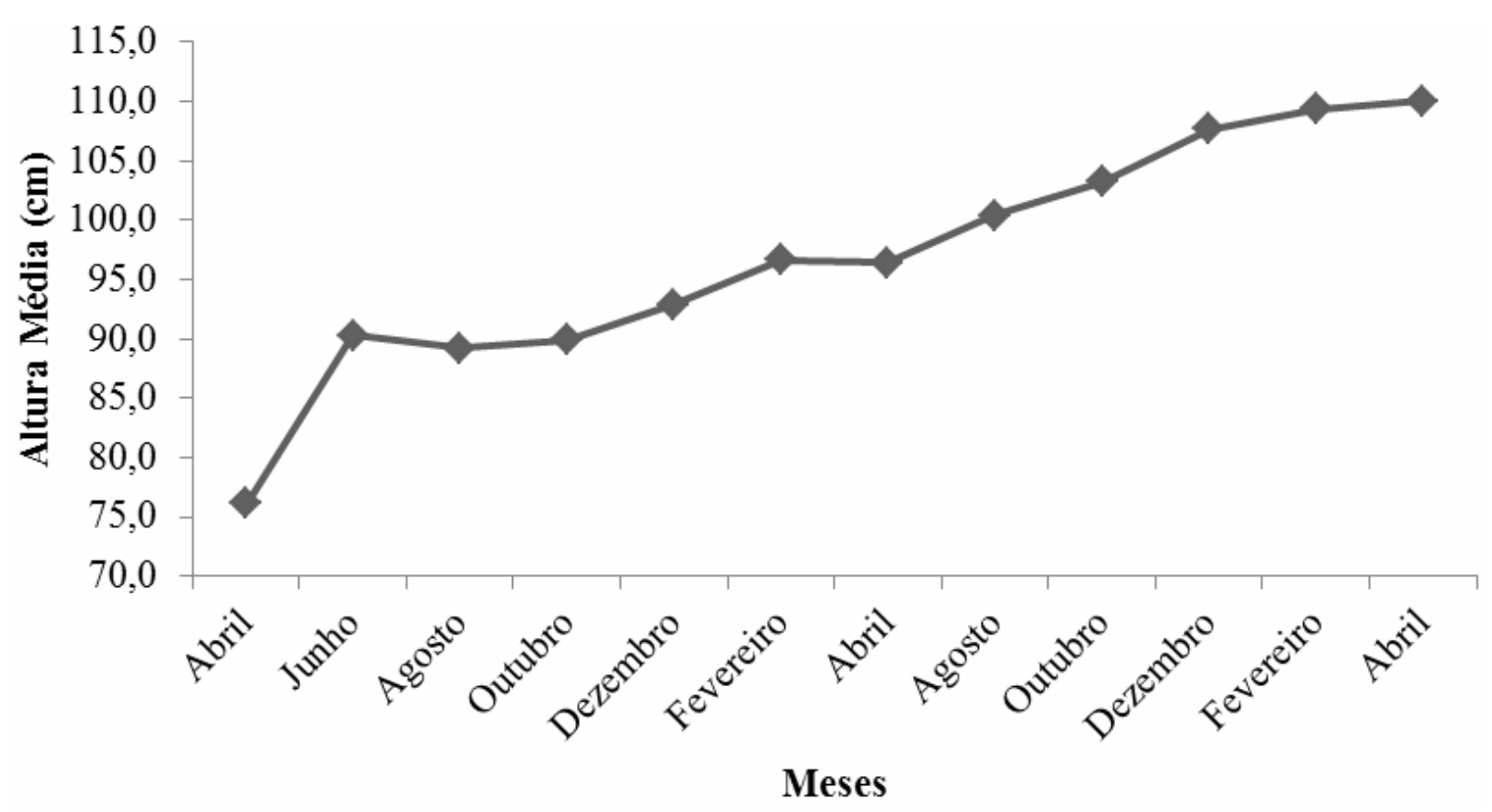

FIGURA 1. Evolução da altura das mudas transplantadas para uma floresta secundária das mudas de Astronium gracile aos 24 meses.

Fonte: Autores (2018).

Apesar de Astronium gracile já ter sido classificada como secundária tardia (SAITER et al., 2011), as mudas de regeneração natural dessa espécie se beneficiaram da abertura de clareiras causadas por exploração florestal na Amazônia brasileira (QUADROS et al., 2013), como também com as condições de luminosidade quando plantadas em clareiras causadas por exploração florestal de impacto reduzido, sendo recomendada para enriquecimento em clareiras formadas pela exploração de impacto reduzido (GOMES et al., 2010). Essa espécie possui aptidão ecológica regular para reflorestamento de áreas de restauração florestal, após a lavra de minério em unidade de conservação na Amazônia (SALOMÃO et al., 2014).

Portanto, a tolerância ao sombreamento moderado durante as primeiras fases de crescimento indicativas que Astronium gracile é uma espécie promissora para o uso em sistemas agroflorestais. Para isso, é necessário que as mudas sejam introduzidas próximas de plantas que possam oferecer condições de suprir a necessidade de sombra nas primeiras fases da vida.

As informações sobre aspectos silviculturais da espécie estudada devem ser levadas em consideração no planejamento do sistema agroflorestal durante a seleção das espécies que serão consorciadas e na decisão do momento mais adequado da introdução no sistema.

\section{CONCLUSÃO}

O presente trabalho reuniu informações sobre os silviculturais de Astronium gracile, desde a germinação até o desenvolvimento inicial das mudas em ambiente florestal. Essas informações reunidas visaram fornecer suporte necessário para o 
uso em sistemas agroflorestais. Quanto aos aspectos germinativos, verificou-se que a espécie possui um alto percentual germinativo e que não houve diferença significativa entre os substratos testados, indicando que os mesmos são eficientes na germinação das sementes em condições ambientais controladas.

A espécie estudada por tolerar sombreamento (leve a moderado) na fase juvenil apresentou desempenho aceitável quanto à sobrevivência e crescimento e reuniu características silviculturais promissoras para 0 uso em sistemas agroflorestais.

\section{REFERÊNCIAS}

BRASIL. Regras para análise de sementes. Brasília: Ministério da Agricultura e da Reforma Agrária: 2009. 395p.

COELHO, F.R.C.; MIRANDA, I.S.; MITJA, D. Conservação das florestas do Projeto de Assentamento Benfica, sudeste da Amazônia. Ciência Florestal, v. 23, n. 1, p.117, $2013 . \quad$ Disponível em: <https://periodicos.ufsm.br/cienciaflorestal/article/view/8435/pdf_1.> DOI: 10.5902/198050988435.

DO VALE, I.; COSTA, L.G.S.; MIRANDA, I.S. Espécies indicadas para a recomposição da floresta ciliar da sub-bacia do rio Peixe-Boi, Pará. Ciência Florestal, v. 24, n. 3, p. 573-582, 2014. Disponível em: https://periodicos.ufsm.br/index.php/cienciaflorestal/article/view/4535. DOI: http://dx.doi.org/10.5902/1980509815736.

FERREIRA, L.S.; CATTANIO, J.H.; JARDIM, M.A.G. Efeito da topografia e da precipitação na florística e na produção de liteira em Caxiuanã, Pará. Revista Árvore, v. 39, n. 6, p.995-1005. 2015. Disponível em: <http://www.scielo.br/scielo.php?script=sci_arttext\&pid=S0100-

67622015000600995\&lng=en\&nrm=iso>. DOI: 10.1590/0100-67622015000600002.

GOMES, J. M.; CARVALHO, J.O.P.; SILVA, M.G.; NOBRE, D.N.V.; TAFFAREL, M. et al. Sobrevivência de espécies arbóreas plantadas em clareiras causadas pela colheita de madeira em uma floresta de terra firme no município de Paragominas na Amazônia brasileira. Acta Amazonica, v.40, n.1, p.171-178, 2010. Disponível em: < https://acta.inpa.gov.br/mobile/fasciculos/40-1/PDF/v40n1a22.pdf>.

GUALBERTO, M.LC.; RIBEIRO, R.B.S.; GAMA, J.R.V.; VIEIRA, D.S. Fitossociologia e potencial de espécies arbóreas. Revista Agroecossistemas, v.6, n.1, p.42-57, 2014. Disponível em: <https://periodicos.ufpa.br/index.php/agroecossistemas/article/view/1593>. DOI: DOI: 10.18542/ragros.v6i1.1593.

KLEIN, D.R.; ANDRADE, M.M.; DERENGOSKI, J.A.; DUARTE, E.; KREFTA, S.M. et al. Aspectos gerais e silviculturais de Cordia americana, Aspidosperma polyneuron, Toona ciliata e Khaya spp. Revista de Ciências Agroveterinárias, v.15, n.2, p. 155164, 2016.2 Disponível em: <http://www.revistas.udesc.br/index.php/agroveterinaria/article/view/22381171152 2016155>. DOI: http://dx.doi.org/10.5965/223811711522016155 
LABOURIAU, L.G. A germinação de sementes. Washington: Organização dos Estados Americanos - Programa Regional de Desenvolvimento Científico e Tecnológico: 1983. (Série Biologia. Monografia.) 24p.

LEMOS, D.A.N.; FERREIRA, B.G.A.; SIQUEIRA, J.D.P.; OLIVEIRA, M.M.; FEREIRA, A.M. Floristic and phytosociology in dense "terra firme" rainforest in the Belo Monte Hydroelectric Plant influence area, Pará, Brazil. Brazilian Journal of Biology, v.75, n.3, suppl.1, p.257-276. 2015, Available from: <http://www.scielo.br/scielo.php?script=sci_arttext\&pid=S1519-

69842015000500257\&lng=en\&nrm=iso $>$. DOI:10.1590/1519-6984.01814BM.

MENDONÇA, G.C.; CHICHORRO, J.F.; MENDONÇA, A.R.; GUIMARÃES, L.A.O.P. Avaliação silvicultural de dez espécies nativas da mata atlântica. Ciência Florestal, v. 27, n. 1, p. 277-290, 2017. Disponível em: http://www.redalyc.org/pdf/534/53450420023.pdf

NIERI, E.M.; MACEDO, R.L.G.; VAZ MARTINS, T.G; MELO, L.A; VENTURIN R.P. et al.Comportamento silvicultural de espécies florestais em arranjo para integração pecuária floresta. Floresta, v.48, n.2, p.195-202, 2018. Disponível em: https://revistas.ufpr.br/floresta/article/view/54744/35339. DOI: 10.5380/rf.v48 i2.54744.

QUADROS, L.C.L.; CARVALHO, J.O.P.; GOMES, J.M.; TAFFAREL, M.; SILVA, J.C.F. Sobrevivência e crescimento de mudas de regeneração natural de Astronium gracile Engl. em clareiras causadas por exploração florestal na Amazônia brasileira. Ciência Florestal, v. 23, n. 3, p. 411-416, 2013. Disponível em: https://periodicos.ufsm.br/cienciaflorestal/article/view/10552/pdf. DOI: $10.5902 / 1980509810552$.

SAITER, F.Z.; DAN, M.L.; THOMAZ, L.D. Floristic and structure of a secondary urban forest with a long history of man-made disturbances in Espírito Santo state, Brazil. Brazilian Geographical Journal: Geosciences and Humanities Research Medium, v.2, n.1, p.69-85. 2011. Disponível em: < http://www.seer.ufu.br/index.php/braziliangeojournal/article/view/11553/7576>.

SALOMÃO, R.P; BRIENZA JÚNIOR, S.; ARAÚJO ROSA, N. A. Dinâmica de reflorestamento em áreas de restauração após mineração em unidade de conservação na Amazônia. Revista Árvore, v. 38, n. 1, 2014. Disponível em: http://www.redalyc.org/html/488/48830661001.

SANTOS, D.R.; SILVA, M.M.; ARAÚJO, A. Diversidade de uso de espécies vegetais presente em florestas primárias e secundárias, em Altamira, Pará. Cadernos de Agroecologia, v.13, n.1, 2018. Disponível em: http://cadernos.abaagroecologia.org.br/index.php/cadernos/article/download/865/413.

SANTOS, S.R.G.; BEZERRA, A.C.F.; OLIVEIRA, P.G.M.; SILVA, S.D.R.; PAULA, R.C. Avaliação de substratos e temperaturas para sementes de espécies florestais. Revista da Universidade Vale do Rio Verde, v. 13, n. 1, p. 66-76, 2015. Disponível em: https://dialnet.unirioja.es/servlet/articulo?codigo=5106746. 
SOUZA, F.B; MENGARDA, L.H.G.; SPADETO, C.; LOPES, J.C. Substratos e temperaturas na germinação de sementes de gonçalo-alves. Revista Trópica: Ciências Agrárias e Biológicas, v. 6, n. 3, p.76-86, 2012. Disponível em: http://www.periodicoseletronicos.ufma.br/index.php/ccaatropica/article/view/905/1031

STOLARSKI, O.C.; GORENSTEIN, M.R.; LUBKE, M.; LUBKE, L.; O'CONNOR, P.H.P. et al. Trema micrantha (L.) Blume. Em plantações para restauração ecológica: desenvolvimento inicial na floresta subtropical brasileira. Ciência Florestal, v.28, n.3, p.1217-1229, 2018.2 Disponível em: https://periodicos.ufsm.br/cienciaflorestal/article/view/33351/pdf. DOI: http://dx.doi.org/10.5902/1980509833351. 